\title{
Forecasting Volatility and Pricing Option: An Empirical Evaluation of Indian Stock Market
}

\author{
Sunaina Kanojia ${ }^{1}$, Neeraj Jain ${ }^{21}$ \\ ${ }^{1}$ DepartmentofCommerce, DelhiSchoolofEconomics, Universityof Delhi, India \\ ${ }^{2}$ DepartmentofCommerce, DelhiSchoolofEconomics, Universityof Delhi, Indian
}

\begin{abstract}
The present study empirically investigates and examine seven models of volatility forecasting, namely unconditional standard deviation (also written as Long Term Moving Volatility), Standard GARCH (Generalized Autoregressive Conditional Heteroscedasticity) model, GJR-GARCH model, Exponential GARCH model (eGARCH), Asymmetric Power GARCH model (apGARCH), Component Standard GARCH model $(\mathrm{cs} G A R C H)$, and Option Implied Volatility model to gauge the most appropriate model of volatility forecasting in Nifty constituent companies. The assessment of risk and determination of price of the asset class is primarily dependent on the volatility calculated for the class of asset. In view of obtaining precision in the process of determining the price of the option and making hedging most effective, it's imperative to have the most appropriate method of calculating the volatility. The present study finds option implied volatility as the best performing model except in few categories of option data where VIX outperformed. Similarly on empirical performance of Black-Scholes $(B S)$ model the present study finds that performance is not same across various maturities which indicate volatility is not constant as assumed by BS model during the tenure of the study in Indian market.
\end{abstract}

Keywords: Black-Scholes model, GARCH Family Models, Standard Deviation, Volatility

\section{Introduction}

One of the fundamental issues within finance is the valuation of future returns and the most proximate valuation of expected return from an asset. While there are various alternatives but a basic paradigm for valuation, in both academic and in practice, is assumption of competitive market equilibrium: The price that will apply in the market is that price which equates total demand to total supply. The Black-Scholes (1973) [3] was the first one who derived the valuation formula for call option rely on the notion of market equilibrium in only the weakest possible sense, known as "arbitrage reasoning." If, under their respective assumptions, the market price does not equate to actual price, then market participants would have an opportunity to create an "arbitrage," that is, to trade securities so as to make unbounded profits with no initial investment and no subsequent risk of loss. Their valuation approach was based on creating a hedged portfolio whose value is completely deterministic over a short span of time (dt). The idea of hedging option with the underlying stock was first coined by Thorp and Kassouf (1967) [14]. But Black and Scholes found that elimination of risk in this way leads to restriction on the relationship between the price of an option and the price of the underlying assets. They found, if portfolio is completely hedged (no risk involved), then expected return on the position must be the riskless interest rate (also as per CAPM). Even though the CAPM does not rely on arbitrage reasoning, it also played a key role in the development of the Black-Scholes formula. Under the assumption that stock follows Geometric Brownian Motion and using Ito Lemma, Black and Scholes lead to following partial differential equation :-

$$
\frac{\partial V}{\partial t}+\frac{1}{2} \sigma^{2} S^{2} \frac{\partial^{2} V}{\partial S^{2}}+r S \frac{\partial V}{\partial S}=r V
$$

where, VisthepriceoftheoptionasafunctionofstockpriceSandtimet,ristherisk-

freeinterestrate,andøisthevolatilityofthestock.Solving the equation subject to boundary condition

$$
c(S, T)=\max \{S-K, 0\}
$$

leads to famous B-S formula for call option $c_{t}$ :-

$$
c_{t}=S_{t} \Phi\left(d_{1}\right)-K e^{-r t} \Phi\left(d_{2}\right), \text { where }
$$

${ }^{1}$ Corresponding Author. E-mail: neeraj2409@outlook.com

DOI: $10.9790 / 487 X-1907010108 \quad$ www.iosrjournals.org $\quad 1 \mid$ Page




$$
\begin{gathered}
d_{1}=\frac{\ln \left(\frac{s_{t}}{K}\right)+\left(r+\frac{\sigma^{2}}{2}\right) \tau}{\sigma \sqrt{\tau}} \\
d_{2}=d_{1}-\sigma \sqrt{\tau}
\end{gathered}
$$

where $c$ ispriceofeuropeancalloption, Kisthestrikepriceand $\tau=(T-t)$ isthe maturityofacalloption.

Many investors and major corporations use these methods for planning, purchasing, pricing, or accounting purposes. In addition, to valuing straight put and call options, corporations use Black-Scholes modeling to value executive stock compensation plans, real production options, warrants, convertible, securities, debt, and so on. (In fact, for many of these applications, the methods are at times applied inappropriately.)

BS model is based on many assumptions. Various authors have tested the performance of BS model in the past. Fischer Black (1972) [2] compared option prices calculated from BS equation with actual market prices. They observed that "the model tends to overestimate the value of an option on a high variance security; market traders tend to underestimate the value, and similarly, while the model tends to underestimate the value of an option on a low variance security, market traders tend to overestimate the value" (Fischer Black, 1972, pg 416-417). Part of the deviations of the observed market prices from the model prices is thought by Black and Scholes, to be due to errors in measurement of the variance of stock returns. The measure of stock return variance used in their study was the sample variance of historic stock returns. Black and Scholes found that a strategy of selling options which are overvalued and buying undervalued options could yield substantial profits. However, when they included the transaction cost of trading in options, they found that the implied profits from this strategy vanished.

MacBeth and Merville (1979) [9] examined daily prices of options on six underlying securities over a one year time period. Their results were exactly opposite to those reported by Black wherein he stated that deep in the money (out of the money) options generally have B-S model prices which are greater (less) than market prices. They proposed that these conflicting empirical observations may, at least in part, be the result of a nonstationary variance rate in the stochastic process generating stock prices. McBeth and Mervile (1979) [9] also suggested trading strategy which involves selling deep in the money options and buying deep out of the money options. Whether or not this strategy yields abnormally high returns were not tested by Beth and Mervile. Lauterbach and Schultz (1990) [7] examined a sample of over 25,000 daily warrant prices to empirically investigate potential problems with the commonly used warrant pricing model proposed by Black and Scholes as an extension of their call option model. They found Black- Scholes cause biases in model prices for almost all warrants and over the entire sample period because of assumption of constant volatility. They further showed that forecast could be better if constant elasticity of variance is used.

\section{Relevance Of The Study}

Among all the variables in the BS model, volatility is most crucial variable in the BS model. Unlike other variables, volatility cannot be observed directly from the market. Therefore, various model has been developed in the past to accurately forecast the volatility and price option accordingly. The present study test the empirical performance of BS model under various volatility models namely, namely Unconditional Standard Deviation (also written as Long Term Moving Volatility), Standard GARCH (Generalized Autoregressive Conditional Heteroscedasticity) model, GJR-GARCH model, Exponential GARCH model (eGARCH), Asymmetric Power GARCH model (apGARCH), Component Standard GARCH model (csGARCH), and Option Implied Volatility model including Volatility Index (VIX). The present study serve two dual objectives, one to test the empirical performance of BS model under various volatility models, and another to test the performance of various volatility models (assuming BS model is correctly specified).

\section{Data Description And Various Input Calculation}

To forecast volatility and evaluate the performance of Black-Scholes models, this study used secondary data of daily closing value of S\&P CNX Nifty Index, daily data of Nifty Option contracts, daily closing value of India Volatility Index (VIX), Nifty future prices, and MIBOR. Details about the data given below in the Table 1:

Table1: Data Description

\begin{tabular}{lcccc}
\hline Series & Frequency & Beginning & End & Observations \\
\hline Nifty & Daily & 1stApr2002 & 31stMar2015 & 3242 \\
VIX & Daily & 1stApr2009 & 31stMar2015 & 1490 \\
NiftyOption & Daily & 1stApr2009 & 31 stMar2015 & 23155 \\
NiftyFuture & Daily & 1stApr2009 & 31 stMar2015 & - \\
MIBOR & Daily & 1stApr2009 & 31 stMar2015 & 1509 \\
\hline
\end{tabular}


The data prior to 1st April 2002 is not considered because trading in Nifty future was introduced on June 4, 2001 and many empirical studies, for example, Raju and Karande (2003)[11], Bandivadekar and Ghosh (2003) [1] and Singh and Kansal (2011)[12] etc. found significant decline in market volatility after the introduction of derivative product especially future and option contracts.

\subsection{Input Calculation}

\subsubsection{Exclusion of Outliers}

Following exclusion criteria has been used on the option pricing data.

1. Expiry:Only those contracts expiring on the same month or immediate next month have been considered for the study. This same exclusion criterion is used by NSE on option contracts to calculate VIX. So, toensure consistency between VIX and other volatility model, all option contracts expiring beyond next immediate month have not been considered in this study. Further, contracts having maturity less than 6 days [trading days] to maturity are excluded. Short term option contracts are very sensitive to the non-synchronous price issues and driven by market sentiments.

2. Moneyness(m): Option moneyness (m) is defined as ratio of future price of underlying security to option strike price.

$$
m=\frac{F(t, \tau)}{K}
$$

where, $F(t, \tau)$ is future price of NIFTY at time $t$ of expiry $\tau$ years and $K$ is the option strike price. Option contracts trading at highly deep in-the-money contains very less information about volatility. Therefore, in the present study highly deep in-the-money option contracts $(m>1.10)$ has not been considered for the empirical investigation. Similarly, option contracts trading at deep-out-money, for $m<0.90$, has not been considered for empirical investigation.

3. Volume: Options having turnover less than 100 Lacs has not been considered. Again this is done to ensure that only actively traded option is considered and closing value of index is synchronized with option closing value.

4. Arbitrage Opportunities: European call option is said to provide arbitrage opportunity, if

$$
c(t, \tau)>\max \left\{(F(t, \quad \tau)-K) e^{-r \tau}, 0\right\}
$$

where, $c(t, \tau)$ is the option price at time $t, F(t, \tau)$ is future price of NIFTY having same maturity as option contract, and $\tau$ is expiry period. So, option contracts not satisfying arbitrage condition have excluded from the study.

Based on these criteria only 23155 observations (approximately $3 \%$ of original samples) were left for analysis in the database. The selected observations were divided into several categories according to their moneyness and maturity. Various categories on the basis of moneyness and number of observations therein have been shown in the Table 2 .

Table 2: Options categories and number of observations

\begin{tabular}{ccc}
\hline Moneyness & Category Name & No. of Observations \\
\hline$m<0.95$ & Deep out of the money (Deep OTM) & 3308 \\
$0.95<m \leq 0.98$ & OTM & 4502 \\
$0.98<m \leq 1.00$ & Near OTM & 3570 \\
$1.00<m \leq 1.02$ & Near In the Money (ITM) & 3362 \\
$1.02<m \leq 1.05$ & ITM & 4122 \\
$1.05<m$ & Deep ITM & 4291 \\
\hline
\end{tabular}

Similarly, on the basis of maturity, contracts have been partitioned into 4 categories: (i) Contracts having maturity less than or equal to 10 days [trading days], (ii) maturity exceed 10 days but less than or equal to 22 days, (iii) maturity exceed 22 days but less than 34 days, and (iv) maturity more than 34 days. To make more comprehensive analysis, all the contracts have further been divided on the basis of both moneyness and maturity. The number of contracts in each subcategory is shown in Table 3.

Table3: Number of observations in each sub option categories

\begin{tabular}{lrcll}
\hline \multicolumn{1}{c}{ Categories } & $\tau \leq 10$ & $10<\tau \leq 22$ & $22<\tau \leq 34$ & $\tau>34$ \\
\hline$m<0.95$ & 97 & 844 & 1611 & 756 \\
$0.95<m \leq 0.98$ & 471 & 1732 & 1684 & 615 \\
$0.98<m \leq 1.00$ & 665 & 1375 & 1130 & 400 \\
$1.00<m \leq 1.02$ & 650 & 1279 & 1048 & 385 \\
$1.02<m \leq 1.05$ & 781 & 1502 & 1354 & 485 \\
$1.05<m$ & 609 & 1842 & 1471 & 369 \\
\hline
\end{tabular}




\subsubsection{Strike Price of the Option}

The exercise or the strike price is the price at which the call option contract gets executed. It is well-known by both the buyer and the seller of the option at the time of entering into the contract.

\subsubsection{Time to Maturity}

Time to maturity is the time period left for the expiration of the option contract. It is expressed in a year. Empirical work suggests that it is trading that cause volatility not the information. Thus, to estimate time to maturity, we have only considered trading days between option trading date and its expiration date. Not only weekend but any other days on which market remain closed is not considered for estimating time to maturity (List of holidays are available on the NSE website and declared in advance).

\subsubsection{Interest Rate}

Black-Scholes (BS) model uses risk-free rate of interest as an input to estimate current option price. The BS formula assumes both lending and borrowing is possible at risk- free interest rate. Use of 91 days treasury rate is primary choice among scholars but it must be noted that borrowing cannot be made at risk-free interest rate until and un- less the investor is government itself. Therefore, instead of using T-Bill, we have used MIBOR as a proxy of interest rate. So, where option maturity is less than 14days [calendar days, not trading] we have used 14 days MIBOR. Where option maturity is between 15 days to 30days, we have used 30days MIBOR rate. 3Months MIBOR is used for Option having maturity more than 30days. Moreover, NSE also uses MIBOR rate to calculate VIX index which uses option data. Thus, the use of MIBOR is not only logical choice but also consistent with the VIX.

\subsubsection{Volatility Estimation}

Volatility forecasted from various models has been used an input into the BS model. But, before using as an input, the daily forecasted volatilities are first converted into annualized volatilities. We multiplied the daily volatility from the square root of number of trading days in a year to get annualized volatility. So,

Annualized Volatility $=$ Daily Volatility $\sqrt{ }$ Number of trading days in year

$$
\begin{aligned}
& =\text { Daily Volatility } \sqrt{248} \\
& =\widehat{\sigma}_{t} \sqrt{248}
\end{aligned}
$$

where,

ôtisthedailyforecastedvolatilityfortimetforecastedat $(t-1)$. Whereas,no adjustmentismadeinVIXbecauseitisalreadyexpressedinannualizedvolatility.

\subsubsection{Future Price}

In our study, we have used Modified Black-Scholes (BS) model which require Future Price (of NIFTY) to compute option price. Future price is directly observable and can be downloaded from NSE website. It doesn't require any adjustment before using in BS model.

\section{Methodology}

To forecast volatility, present study considered seven models, namely Unconditional Standard Deviation (also written as Long Term Moving Volatility), Standard GARCH (Generalized Autoregressive Conditional Heteroscedasticity)(see Bollerslev, 1986) [4] model, GJR-GARCH model (see, Glosten,Jagannathan, andRunkle, 1993) [6], Exponential GARCH model (eGARCH) (see, Nelson, 1991) [10], Asymmetric Power GARCH model (apGARCH) (see, Ding,Granger,andEngle, 1993; Taylor, 2012)[5] [13], Component Standard GARCH model (csGARCH) (see, LeeandEngle,1993) [8] , and Option Implied Volatility model (see, BlackandScholes,1973) [3].

\subsection{Forecasting Volatility}

Volatility for NSE Nifty 50 from all models of volatility are forecasted, period from 1st April 2009 to 31st March 2015 (Backtesting Period) using in-sample-period (estimation period) of 1st April 2002 to 31st March 2009, on rolling basis. It involve re-estimating the parameters of the model as more recent data become available. The parameters of every GARCH-family models have been updated after every 15 days [trading days] and for historical volatility models parameters are revised for each trading day. For example, first, model parameters are estimated using in-the-sample data, period from 1st April 2002 to 31st March 2009, then forecasting is made for next 15 trading days ie from 1st April 2009 to 22nd April 2009 (include only 15 trading days). After that in- the-sample period data is forwarded by 15 trading days from behind (now in-the-sample period span from 22nd April 2002 to 22nd April 2009) and parameters are re-estimated including recent observations, ensuring that number of observations remain same every time in-the-sample period. Now, again forecasting will be made for next 15 trading days and procedure will be repeated till the entire out-of-sample 
period is covered. Similar procedure has been followed for every GARCH-Family model for forecasting volatility.

\subsection{Performance Evaluation}

Three loss functions are being used to assess the performance of BS model with different volatility models.

1. Root Mean Square Error (RMSE)

$$
R M S E=\sqrt{\frac{1}{N} \sum_{t=1}^{N}\left(\widehat{c_{t}}-c_{t}\right)^{2}}
$$

2. Mean Absolute Error (MAE)

$$
M A E=\frac{1}{N} \sum_{t=1}^{N}\left|\widehat{c_{t}}-c_{t}\right|
$$

3. Mean Absolute Percent Error (MAPE)

$$
\text { MAPE }=\frac{1}{N} \sum_{t=1}^{N} \frac{\left|\widehat{c_{t}}-c_{t}\right|}{c_{t}}
$$

Where $c t$ istheactualcalloptionpriceand $\hat{t} t$ isBSmodelpriceforcalloption.

Thehigherthevalueofthestatistics, worstistheperformanceofthemodel.

\section{Analysis And Results}

The analysis of the study reveals the mean absolute error, mean absolute percent error, and root mean square error for difference between actual call option price and model price which is examined to determine the most sought after model to estimate volatility of the option in Indian stock market.

\subsection{Moneywise Performance}

Table4: Mean Absolute Error by Moneywise for Option Price

\begin{tabular}{lcccccc}
\hline \multicolumn{1}{c}{ Model } & $m<0.95$ & $0.95<m \leq 0.98$ & $0.98<m \leq 1.00$ & $1.00<m \leq 1.02$ & $1.02<m \leq 1.05$ & $m>1.05$ \\
\hline sGARCH & 19.02339 & 18.22642 & 19.59589 & 18.455635 & 17.214508 \\
gjrGARCH & 19.74524 & 19.61175 & 21.12412 & 20.019929 & 18.484276 \\
eGARCH & 21.16267 & 21.43449 & 22.79687 & 21.498984 & 19.245715 \\
csGARCH & 17.82532 & 17.23180 & 18.94941 & 18.277061 & 14.46348 \\
apARCH & 21.57316 & 21.36936 & 22.57701 & 21.145122 & 19.092332 \\
LTMV & 42.68346 & 63.02125 & 68.92104 & 64.732335 & 49.142405 \\
IV & 10.37281 & 8.55084 & 8.10675 & 8.086648 & 14.49146 \\
VIX & 18.14773 & 16.87600 & 15.26421 & 11.525228 & 6.840104 & 10.276568 \\
\hline
\end{tabular}

Table5: Mean Absolute Percent Error by Moneywise for Option Price

\begin{tabular}{lcccccc}
\hline \multicolumn{1}{c}{ Model } & $m<0.95$ & $0.95<m \leq 0.98$ & $0.98<m \leq 1.00$ & $1.00<m \leq 1.02$ & $1.02<m \leq 1.05$ & $m>1.05$ \\
\hline sGARCH & 0.5770461 & 0.3424147 & 0.1967293 & 0.1062995 & 0.06279494 & 0.0338851 \\
gjrGARCH & 0.6299252 & 0.3744518 & 0.211188 & 0.1150277 & 0.067232 & 0.035007 \\
eGARCH & 0.7115772 & 0.4205594 & 0.2314673 & 0.1256188 & 0.07052184 & 0.0346921 \\
csGARCH & 0.5306338 & 0.3154472 & 0.1859678 & 0.1042496 & 0.06422492 & 0.0350937 \\
apARCH & 0.717253 & 0.4160338 & 0.2286952 & 0.1231391 & 0.06992369 & 0.0350469 \\
LTMV & 1.9685123 & 1.7924982 & 0.9518303 & 0.4447979 & 0.19562611 & 0.0687578 \\
IV & 0.362087 & 0.1864304 & 0.0911411 & 0.0481359 & 0.03569996 & 0.0245494 \\
VIX & 0.6891325 & 0.4052397 & 0.2031343 & 0.0805774 & 0.02512093 & 0.0122087 \\
\hline
\end{tabular}

Table 4 shows mean absolute error for Black-Scholes (BS) model for different volatility models. Analysis of Table 4 revealed that Mean Absolute Error (MAE) is minimum when implied volatility is used as an input into the model for option with moneyness less than 1.02. For option with moneyness more than 1.02, MAE is minimum for Volatility Index (VIX). Results show no single model produce minimum MAE for all categories of option. Long Term Volatility Model (LTMV) is worst model for pricing option as it produces highest MAE for all categories of option. If moneyness-wise absolute errors are analysed, then, for Deep out-ofmoney (OTM) option ( $<$.95), Implied Volatility leads to minimum error and LTMV model leads to maximum error by applying the BS model. In the GARCH family models, csGARCH has minimum MAE for Deep OTM but MAE is far away from the IV model. For example, for Deep OTM, MAE for Implied Volatility (IV) model is 10.373 whereas it is 17.8253 for csGARCH. For OTM options $(.95<\mathrm{m} \leq 0.98)$, again IV model is best performer followed by VIX and LTMV model is worst model. For Near ITM and OTM options, again IV 
model is best performer and LTMV model is the worst model. But for option ITM $(1.02<\mathrm{m} \leq 1.05)$ and Deep in-the-money ( $m>1.05)$, MAE is minimum when VIX is used as an input for volatility in the model. In GARCH family model, MAE is minimum for standard GARCH model for same moneyness but still far away from the best performer. For example, MAE is for Deep in-the-money $(\mathrm{m}>1.05)$ for VIX is 5.1879 while it is 13.96 by sGARCH.

Talking in terms of the percentage errors, shown in Table 5, that Implied Volatility model lead to minimum Mean Absolute Percent Error (MAPE) for all categories of option out-of-money $(\mathrm{m}<=1)$ and near inthe-money (ITM). But for option ITM $(1.02<\mathrm{m}<1.05)$ and deep in-the-money $(\mathrm{m}>1.05)$, MAPE is minimum when VIX is used as an input for volatility in the model. MAPE gets reduce for all the volatility models as option moves in the money. For example, MAPE for VIX for options deep out-of-money (OTM) is $68.91 \%$ while it reduces to $1.2 \%$ for Option Deep ITM. Such consistent improvement in the performance of the model indicates towards the biasness in the volatility. Empirical studies suggest smile pattern in the volatility ie market participants do not use same volatility for price options having distinct moneyness. Again, LTMV model is the worst performer model. In GARCH family model, csGARCH is best performer for all categories of option out of money $(\mathrm{m}<=1)$ and near in the money. But for option ITM $(1.02<\mathrm{m}<1.05)$ and Deep in the money $(\mathrm{m}>$ 1.05), MAPE is minimum when sGARCH model is used as an input for volatility in the model. But still, the performance of GARCH family model are far away from the overall best performer model. For example, MAPE for sGARCH model for option deep ITM is $3.39 \%$ while it is $1.2 \%$ for best performer model ie VIX. Similarly, MAPE for csGARCH model for option deep OTM is 53.06\% (lowest among GARCH family model) while it is $36.21 \%$ for IV model (best performer model).

Table6: Root Mean Square Error by Moneywise for Option Price

\begin{tabular}{lrrrrrr}
\hline Model & $m<0.95$ & $0.95<m \leq 0.98$ & $0.98<m \leq 1.00$ & $1.00<m \leq 1.02$ & $1.02<m \leq 1.05$ & \multicolumn{1}{c}{$m>1.05$} \\
\hline sGARCH & 32.07427 & 31.08861 & 33.40886 & 31.34922 & 30.215612 & 24.772608 \\
gjrGARCH & 29.28762 & 31.43858 & 34.12968 & 32.18549 & 30.41045 & 23.65111 \\
eGARCH & 29.46172 & 32.69246 & 35.54282 & 33.53034 & 31.065594 & 23.344015 \\
csGARCH & 30.76778 & 30.17689 & 32.91079 & 31.21013 & 30.447865 & 25.039095 \\
apARCH & 30.5398 & 32.85055 & 35.2818 & 33.08532 & 31.079577 & 23.732349 \\
LTMV & 50.23246 & 71.68611 & 78.03052 & 73.95974 & 58.095272 & 35.183048 \\
IV & 17.73215 & 15.49185 & 15.57933 & 14.37952 & 16.386855 & 16.158365 \\
VIX & 21.16113 & 19.27341 & 17.665 & 14.15589 & 9.537102 & 8.493018 \\
\hline
\end{tabular}

Similar results were found when Root Mean Square Error (RMSE) is used and exhibited in Table 6. RMSE penalize higher deviations more than smaller deviation. For all categories of OTM options $(\mathrm{m}<1)$, RMSE is lower for Implied Volatility (IV) model, while for all categories of option ITM (m > 1), RMSE is lower for VIX. Again, as option moves in-the-money, RMSE gets reduce for all model of volatility. LTMV model is the worst performer model.

In conclusion, it seems IV model in absolute and percentage terms is the best input for the BS to price $\mathrm{OTM}(\mathrm{m}<1)$ and Near ITM call option, while VIX is best input to price call option $\operatorname{ITM}(1.02<\mathrm{m}<=1.05)$ and deep in the money $(\mathrm{m}>1.05)$. Further, in the absence of VIX, IV model is best performing model. The performance of no other model is near to implied volatility.

\subsection{Maturity Wise Performance}

Table 7 shows the Mean Absolute Error (MAE) for option price for various volatility models. Options has been categorized on the basis of maturity. Analysis of results from Table 7 shows that in absolute terms the pricing errors are minimized only when the Implied Volatility (IV) is used as an input into the model for all the maturities. Absolute error increases as maturity increase for all the models.

Table7: Mean Absolute Error by Maturity-wise for Option Price

\begin{tabular}{lcccc}
\hline Model & $\tau \leq 10$ & $10<\tau \leq 22$ & $22<\tau \leq 34$ & $\tau>34$ \\
\hline sGARCH & 9.00547 & 15.82900 & 21.32408 & 21.83351 \\
gjrGARCH & 8.87427 & 16.91885 & 22.85958 & 23.51759 \\
eGARCH & 9.22868 & 17.73469 & 24.06581 & 26.32541 \\
csGARCH & 8.77164 & 15.57066 & 21.04249 & 21.12785 \\
apARCH & 9.45823 & 17.57599 & 24.25334 & 25.65870 \\
LTMV & 30.48937 & 45.47842 & 59.95653 & 74.23707 \\
IV & 5.69138 & 6.93594 & 12.03663 & 11.82440 \\
VIX & 8.98515 & 9.55415 & 13.73286 & 17.30963 \\
\hline
\end{tabular}

For example, MAE for IV model when maturity is less than 10 is 5.6914 while it increases to 11.824 when maturity exceeds 34 days. It shows the ability of the Black-Scholes (BS) model to price near the maturity 
option more efficiently than options far away the maturity. As expected, LTMV volatility is the worst performer. csGARCH is second best model for option having maturity less than 10 days, otherwise, VIX is second best model for all maturities.

Table8: Mean Absolute Percent Error by Maturity-wise for Option Price

\begin{tabular}{lrccc}
\hline Model & $\tau \leq 10$ & $10<\tau \leq 22$ & $22<\tau \leq 34$ & $\tau>34$ \\
\hline sGARCH & 0.1584925 & 0.2054765 & 0.2312739 & 0.2374589 \\
gjrGARCH & 0.1555284 & 0.2230333 & 0.2598614 & 0.2524659 \\
eGARCH & 0.1651872 & 0.2422947 & 0.2900882 & 0.3024035 \\
csGARCH & 0.1497268 & 0.1924197 & 0.2173515 & 0.2198504 \\
apARCH & 0.1693011 & 0.2402641 & 0.2917153 & 0.2923268 \\
LTMV & 0.6400195 & 0.8072769 & 0.9658643 & 1.1778748 \\
IV & 0.1019059 & 0.1067696 & 0.1336625 & 0.1390977 \\
VIX & 0.1970614 & 0.2013772 & 0.2465923 & 0.2784894 \\
\hline
\end{tabular}

Talking in terms of the percentage errors (results are shown in Table 8), Implied Volatility (IV) model lead to minimum Mean Absolute Percent Error (MAPE) for all categories of option. Again LTMV model is worse performing model. Interestingly, second best model after IV model is csGARCH for all categories of maturity. Further, the difference in the performance of various GARCH family model is not quite large. For example, MAPE for csGARCH model for options maturity less than 10 days is $14.97 \%$ while it is $15.85 \%$ for sGARCH model, and $15.55 \%$ for gjrGARCh model. Implied volatility performs best even when RMSE is used. Results for RMSE are shown in Table 9.

Table9:RootMeanSquareErrorbyMaturity-wiseforOptionPrice

\begin{tabular}{lcccc}
\hline Model & $\tau \leq 10$ & $10<\tau \leq 22$ & $22<\tau \leq 34$ & $\tau>34$ \\
\hline sGARCH & 17.19043 & 27.040732 & 36.00372 & 33.83928 \\
gjrGARCH & 15.54556 & 27.482031 & 34.85922 & 35.48726 \\
eGARCH & 15.7422 & 27.937075 & 35.5245 & 37.85916 \\
csGARCH & 17.28807 & 26.537485 & 35.81926 & 32.71561 \\
apARCH & 16.38775 & 27.753126 & 36.07599 & 37.28117 \\
LTMV & 37.4675 & 54.337281 & 69.25374 & 83.77617 \\
IV & 10.55271 & 9.871307 & 21.5354 & 16.91426 \\
VIX & 12.58853 & 11.952443 & 17.32507 & 21.5012 \\
\hline
\end{tabular}

It seems that Implied Volatility (IV) model is the best input for Black-Scholes (BS) model to price call option for all maturities of option. But each category of maturity has mixture of option with varying moneyness. As we have seen in the results of previous section, IV model performed best only for option out-of-money and near in-the-money (ITM) options, whereas, Volatility Index (VIX) was the best performer for pricing ITM (1.02 $<\mathrm{m}<=1.05)$ and deep ITM $(\mathrm{m}>1.05)$ option.

\section{Conclusion}

The present study finds option implied volatility as the best performing model except in few categories of option data where VIX outperformed. Among GARCH family models only, there is not a single model which consistently leads to minimized percentage and absolute error for all categories of option. Component sGARCH model is the best model for pricing either out-of-money option or near ITM option, whereas, sGARCH is best performing model for pricing option out of money. Overall, no single model perform best for all categories of options. Results found long term moving volatility (an unconditional measure of volatility), most widely used measure of volatility, as a worst model among all category of models. These results are consistent with the past studies that favour implied volatility for pricing option rather than GARCH derived volatility. The effectiveness of hedging primarily depend on accuracy of volatility forecasting. Therefore, the study recommend the use of implied volatility to hedge market risk effectively. The use of implied volatility also serve another advantage. It does not require the entire past history of option data to forecast volatility unlike GARCH models. It is pertinent to note that applying the best performing model to forecast volatility and thereafter pricing leads to close approximation of value of underlying asset which is worthy to be followed by both investors and institutions involved in financial markets. Empirical performance of BS models found that performance is not same across various maturities that indicate volatility is not constant as assumed by BS model. Further, results show systemic decline in MAE and MAPE for all volatility model as option moves into the money and as maturity decrease. This shows BS model is more efficient in estimating price of options which are near at-the-money and 
near the maturity. In this way, present study provide rationale for using more advanced model for pricing options and cautious to the investors who use BS model to price options.

\section{References}

[1] S. Bandivadekar and S. Ghosh, Derivatives and volatility on Indian stock markets, Reserve Bank of India Occasional Papers, 24(3), 2003, 187-201.

[2] F. Black and M. Scholes, the Valuation of Option Contracts and a Test of Market Efficiency, The Journal of Finance, 27(2), 1972, 399-417.

[3] F. Black and M. Scholes, The pricing of options and corporate liabilities, The journal of political economy, 1973, 637-654

[4] T. Bollerslev, Generalized autoregressive conditional heteroscedasticity, Journal of econometrics, 31(3), $1986,307-327$.

[5] Z. Ding, C. W. Granger, and R.F. Engle, A long memory property of stock market returns and a new model,Journal of empirical finance, 1(1), 1993, 83-106.

[6] L.R. Glosten, R. Jagannathan, and D.E. Runkle, On the relation between the expected value and the volatility of the nominal excess return on stocks, The journal of finance, 48(5), 1993, 1779-1801.

[7] B. Lauterbach, and P. Schultz, Pricing Warrants: An Empirical Study of the Black-Scholes Model and Its Alternatives, The Journal of Finance, 45(4),1990, 1181- 1209.

[8] G. Lee and R.F. Engle, A permanent and transitory component model of stock return volatility, 1993, Available at SSRN 5848.

[9] J.D. MacBeth. and L.J. Merville, An Empirical Examination of the Black- Scholes Call Option Pricing Model,The Journal of Finance, 34(5), 1979, 1173- 1186.

[10] D.B. Nelson, Conditional heteroskedasticity in asset returns: A new approach, Econometrica: Journal of the Econometric Society, 1991, 347-370.

[11] M.T. Raju and K. Karande, Price discovery and volatility on NSE futures market, SEBI Bulletin, 1(3), 2003, 5-15.

[12] G. Singh and S. Kansal, Impact of Derivative Trading on Stock Market Volatility during Pre and Post F\&O Period: A Case Study of NSE, Management Convergence, 1(1), 2011, 1-10.

[13] S.Taylor, Financial returns modelled by the product of two stochastic processes, a study of daily sugar prices,Edward Elgar, 2012, 441-464.

[14] E.O. Thorpand S.T. Kassouf, Beat the market, New York: Random, 1967. 